\title{
Essential role of Fbxl14 ubiquitin ligase in regulation of vertebrate axis formation through modulating Mkp3 level
}

Cell Research (2012) 22:936-940. doi:10.1038/cr.2012.37; published online 13 March 2012

\section{Dear Editor,}

The F-box family of proteins (FBPs), the substraterecognition components of the Skp1-Cul1-F-box-protein (SCF) ubiquitin ligase, are evolutionarily conserved in various species and exhibit key roles in a wide array of biological processes, including tumorigenesis and development [1-3]. Although approximately 70 FBPs have been identified in humans $[4,5]$, only a few of them have been well characterized. Fbxl14 (F-box and leucine-rich repeat protein 14) has been reported to regulate Snail2 protein during neural crest development of Xenopus laevis embryos [6] and to control Snaill protein stability in mammalian cells [7]. There are two homologs of Fbx114 in zebrafish, namely Fbxl14a and Fbxl14b. In this study, we show that Fbxl14a and Fbxl14b are differential regulators of the dorsoventral (DV) patterning of zebrafish embryos and further demonstrate that their roles in axis formation are differentially executed through the modulation of MAP kinase phosphatase-3 (Mkp3) ubiquitination and protein levels.

As a first step to exploring the role of Fbxl14a and Fbxl14b during zebrafish development, we analyzed the expression patterns of the two $f b x l 14$ genes by RT-PCR and whole-mount in situ hybridization (Supplementary information, Data S1 and Table S1). $f b x l 14 a$ mRNA is maternally provided and was expressed during all of the investigated stages. In contrast, $f b x l 14 b$ is mainly zygotically activated, and its expression was first notable at the dome stage, approximately $4.3 \mathrm{~h}$ post-fertilization (hpf) (Supplementary information, Figure S1).

To investigate the role of $f b x l 14 a$ and $f b x l 14 b$ in the embryonic development, we first injected zebrafish embryos with synthetic mRNAs corresponding to these two genes (Supplementary information, Data S1). The injection of $f b x l 14 a$ mRNA caused no observable phenotype (Figure 1A, b and d), whereas injection with the same amount of $f b x l 14 b$ mRNA caused as many as $79 \%$ of the embryos to be dorsalized. Compared with the control embryos (Figure 1A, a and e), those injected with the $f b x l 14 b$ mRNA exhibited an abnormal elongated shape at $11 \mathrm{hpf}$ (Figure 1A, c) and characteristic phenotypes of embryonic dorsalization by $24 \mathrm{hpf}$ (Figure 1A, f-j). We also showed that murine $f b x l 14$ mRNA is able to dorsalize zebrafish embryos in a similar fashion to $f b x l 14 b$, but not $f b x l 14 a$, suggesting an evolutionarily conserved role for $f b x l 14$ in axis patterning (Figure 1C).

To elucidate the functions of endogenous $f b x l 14 a$ and $f b x l 14 b$, morpholino oligonucleotide (MO)-mediated gene knockdown was then employed (Supplementary information, Data S1). The effectiveness of the MOs was tested by co-injecting a morpholino with the reporter plasmid $f b x l 14 a(b)-5$ 'UTR-GFP (Supplementary information, Figure S2). Embryos injected with $A$-MO exhibited weakly dorsalized phenotypes: premature detachment of the tailbud from the yolk at approximately the five-somite stage (Figure 1B, 1), in contrast to the tight attachment of the tailbud around the yolk in the control embryos (Figure 1B, k) and a partial loss of the caudal ventral fin at $24 \mathrm{hpf}$, which are characteristic of weakly dorsalized phenotypes ( $\mathrm{C} 1$ and $\mathrm{C} 2$ classes) (Figure 1B, o). In contrast, embryos injected with $B$-MO displayed epiboly defects and a shorter anterior-posterior axis at early stages (Figure 1B, m); by $24 \mathrm{hpf}$, these embryos were often reduced in length and girth, with a variably reduced head and eyes and an edema posterior to the yolk extension at the site of blood formation (Figure 1B, p). Moreover, the MO-induced phenotypes could be partially neutralized by the co-injection of the corresponding mRNA (Supplementary information, Figure S3), suggesting that $A$-MO and $B$-MO specifically target $f b x l 14 a$ and $f b x l 14 b$, respectively.

Next, we carried out an in situ analysis. The expression alteration of marker genes confirmed our observation, with the dorsal marker gene ( $g s c$ and $c h d$ ) expression domain expanded and ventral marker gene (evel) expression decreased corresponding to dorsalized phenotypes, and the opposite alteration occured to ventralized phenotypes (Supplementary information, Figure S4). These results demonstrate that the functions of $f b x l 14 a$ and $f b x l 14 b$ are unexpectedly distinct from each other. Fbx114a is a weak dorsalizing inhibitor, whereas Fbx114b 
A a

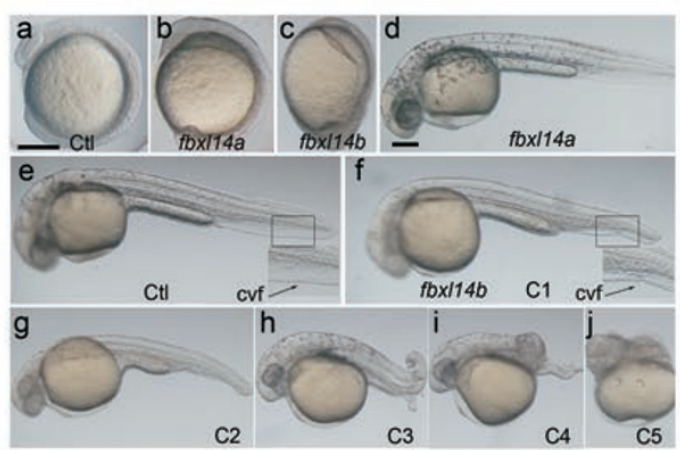

B

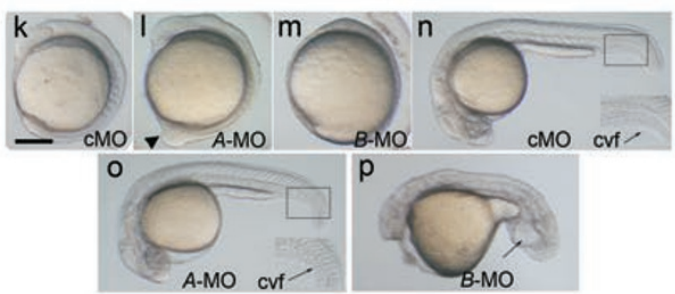

C

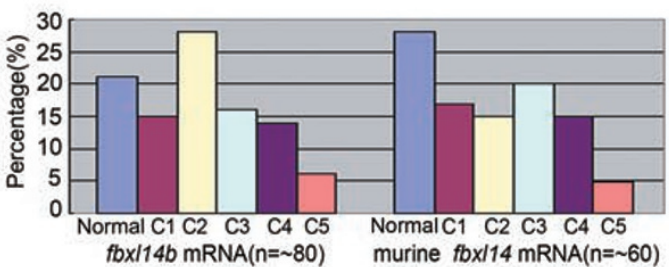

D
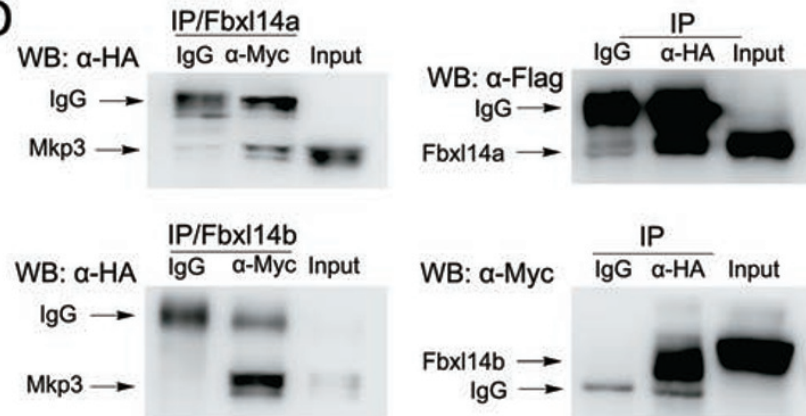
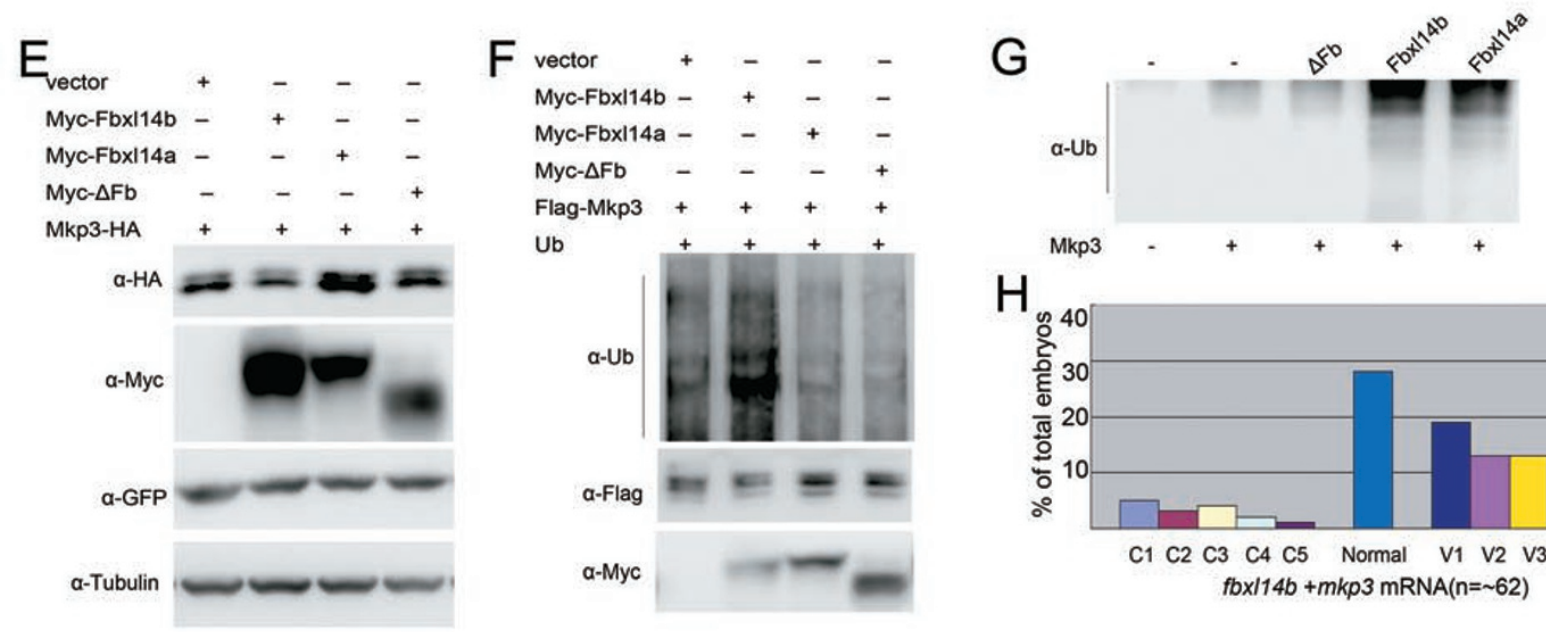

Ub
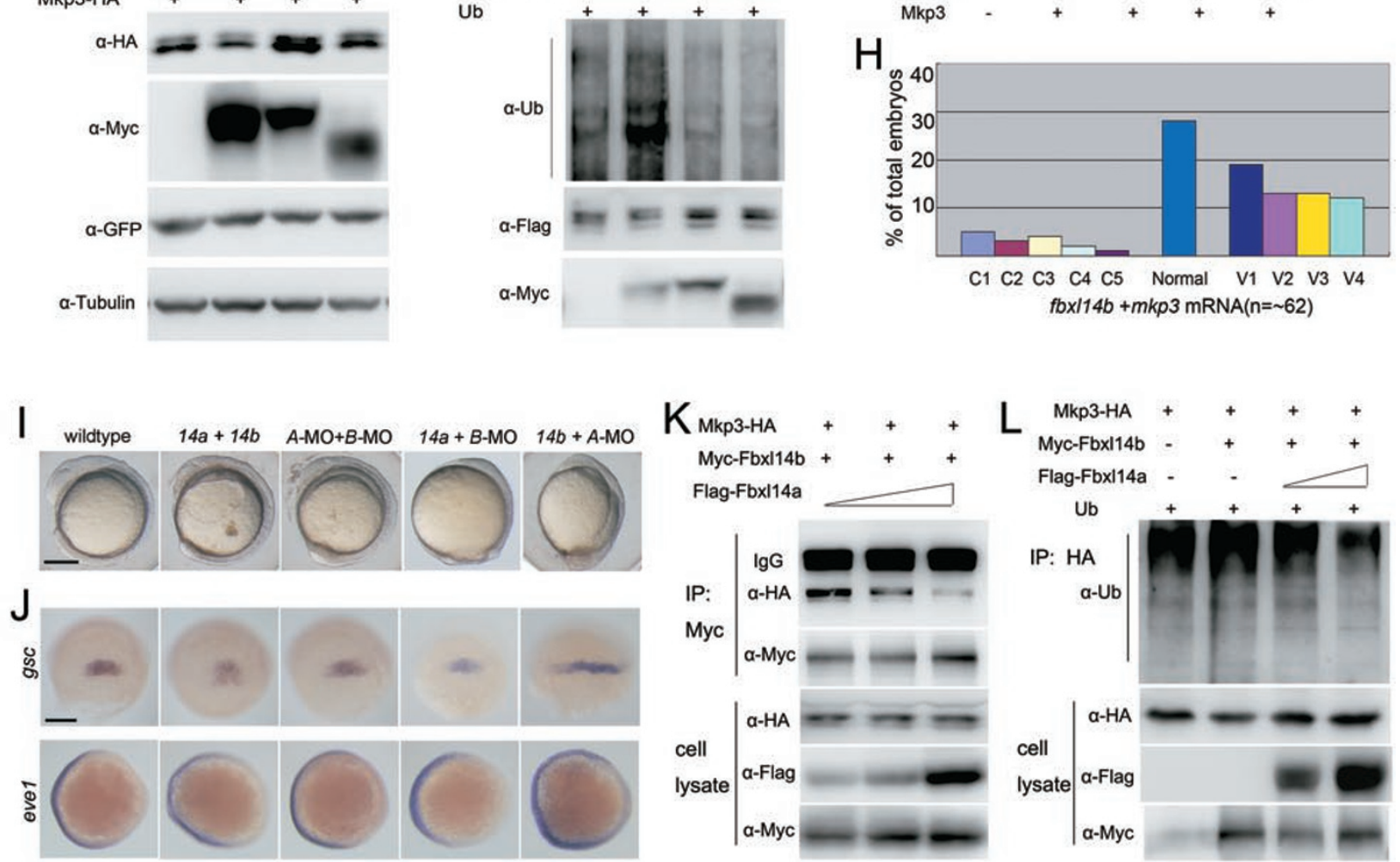

exhibits dorsalizing activity.

To determine which region is responsible for the functional difference between the two homologs, we created

a series of modified forms of the genes (Supplementary information, Figure S5A) and analyzed the effect of their ectopic expression on embryonic development (Supple- 
mentary information, Figure S5B). Injection with $A F$ $+B L$ mRNA, a composite of the F-box domain coding sequence of $f b x l 14 a$ and the LRR coding sequence of $f b x l 14 b$, led to dorsalized phenotypes, although the phenotypic defect was, to some extent, reduced compared to the $f b x l 14 b$ mRNA-injected embryos. In contrast, the embryos injected with $B F+A L$ mRNA, a composite of the F-box domain coding sequence of $f b x l 14 b$ and the LRR coding sequence of $f b x l 14 a$, appeared largely normal, with only $3 \%$ and $4 \%$ of the embryos exhibiting ventral and dorsal defects, respectively. We also analyzed the expression of corresponding markers and confirmed the effect of these mutants (Supplementary information, Figure S5C). Together, these findings support an essential role of the LRRs in Fbxl14b for its dorsalizing activity.
To find candidate substrates of Fbx114b, we first evaluated several ventralizing factors through immunoprecipitation (IP) with Fbx114b (Supplementary information, Data S1). Mkp3, which is involved in the determination of axial patterning of the early zebrafish embryo [8], was identified as an Fbxl14b-binding protein (Figure 1D). Moreover, we found that Mkp3 also interacts with Fbxl14a (Figure 1D). We then investigated the subcellular localization of Fbx114a, Fbx114b and Mkp3. Immunostaining in HeLa cells revealed that all of the three proteins were predominantly detected in the cytoplasm (Supplementary information, Data S1). The Mkp3 expression overlapped well with that of both Fbxl14a and Fbx114b (Supplementary information, Figure S6). Thus, Mkp3 is a binding partner of Fbx114a and Fbx114b.

To examine whether Fbx114b affects the protein level

Figure $1 \mathrm{Fbxl14a}$ and Fbxl14b regulate zebrafish axis formation through differentially modulating Mkp3 level. (A, B) Effects of $f b x / 14 a$ and $f b x / 14 b$ overexpression $(\mathbf{A})$ and knockdown (B). Lateral view of live embryos at five-somite stage (a-c, k-m), $33 \mathrm{hpf}(\mathbf{d}-\mathbf{j})$ or $24 \mathrm{hpf}(\mathbf{n}-\mathbf{p})$. (a, e) Uninjected control embryos. (e) A caudal ventral region was enlarged in the inset so that the caudal ventral fin (cvf) can be seen (same for other pictures). (b, d) Embryos injected with 300 pg of fbx/14a mRNA exhibited no obvious abnormality. (c, f-j) Embryos injected with 300 pg of fbx/14b mRNA displayed an ovoid shape (c) and were morphologically dorsalized (loss of cvf, truncated posteriorly or the trunk tissue is twisted above the yolk) and the dorsalized embryos were sorted into five classes (C1-C5) (f-j). The ratios of the embryos from class 1 to class 5 are $15 \%, 28 \%, 16 \%$, $14 \%$ and $6 \%$, respectively. C1-C5 represent dorsalized phenotypes of increasing strength, the phenotype of which is based on the degree to which the notochord, somites and tail are affected. (k, n) Embryos injected with control MO. (I, m, o, p) Effects of injection of $f b x / 14 a$ and $f b x / 14 b$ MOs into wild-type embryos. Embryos injected with 2.0 pmol A-MO showed early tail protrusion (arrowhead) at five-somite stage (I) and a loss of cvf at $24 \mathrm{hpf}$ (o). Embryos injected with 1.0 pmol $B$-MO showed a developmental retardation $(\mathbf{m})$ and exhibited ventralized phenotypes, including a variably reduced head and eyes and an edema (arrow) posterior to the yolk extension at the site of blood formation (p). Scale bar, $200 \mu \mathrm{m}$. (C) The ratios of dorsalized embryos from C1-C5, caused by injection of $300 \mathrm{pg}$ of $f b x / 14 b$ or $300 \mathrm{pg}$ of murine fbx/14 mRNA, respectively. (D) Mkp3 is coprecipitated with Fbxl14a and Fbxl14b. HEK293T cells were transfected with expression constructs for Mkp3-HA with Myc-Fbxl14a (or Fbxl14a-Flag) and Myc-Fbxl14b, respectively. At $30 \mathrm{~h}$ post-transfection, cells were lysed, and Myc-Fbxl14a or Myc-Fbxl14b was precipitated with anti-Myc antibody. Cell lysates after IP and precipitated complexes were analyzed by immunoblotting using anti-HA antibody for the detection of Mkp3-HA. Then reciprocal IP was carried out. (E) FbxI14a and Fbxl14b increases and decreases the levels of Mkp3 in HEK293T cells, respectively, as determined by western blotting. An empty vector and Myc- $\Delta \mathrm{Fb}$ were used as controls. GFP was used as a transfection control and $\alpha$-tubulin was used as a loading control. (F) Fbxl14a and Fbxl14b regulate the ubiquitination levels of Mkp3 differentially in vivo. HEK293T cells were transfected as indicated for $24 \mathrm{~h}$ followed by treatment with MG132 $(10 \mu \mathrm{M})$ for $6 \mathrm{~h}$. Whole cell extracts were then processed for IP analysis of ubiquitinated Mkp3. (G) Fbxl14b stimulates Mkp3 ubiquitination more significantly than Fbxl14a in an in vitro ubiquitin conjugation assay. Purified GST-fused Mkp3 proteins were incubated with the intact SCF ${ }^{\text {Fbx14 }}$ E3 ligase complexs, which were purified by IP from transfected cells with beads conjugated with anti-Myc antibody. (H) Frequencies of phenotypic classes at $24 \mathrm{hpf}$ of the embryos coinjected with $f b x / 14 b$ and mkp3 mRNAs. Injection doses: 200 pg of fbx/14b mRNA and $200 \mathrm{pg}$ of $m \mathrm{kp} 3$ mRNA. The frequency of phenotypic classes at $24 \mathrm{hpf}$ of the embryos injected with fbx/14b mRNA is shown in Figure 1C. V1-V4 represents ventralized embryos of increasing strength [8]. (I) Morphology of live embryos at the fivesomite stage after indicated injections. Scale bar, $200 \mu \mathrm{m}$. (J) Expression of the marker genes (indicated at the left) at shield stage. Dorsal views with the animal pole oriented toward the top for gsc; animal pole views with dorsal oriented toward the right for eve1. Injection doses: $300 \mathrm{pg}$ of $f b x / 14 a+300 \mathrm{pg}$ of fbx/14b mRNA; $0.8 \mathrm{pmol} A-\mathrm{MO}+1.0 \mathrm{pmol} B-\mathrm{MO} ; 300 \mathrm{pg}$ of fbxl14a mRNA + 1.0 pmol B-MO; 300 pg of fbxl14b mRNA + 1.0 pmol A-MO. Scale bar, $200 \mu \mathrm{m}$. (K) Fbxl14a attenuates the interaction of Fbxl14b with Mkp3. HEK293T cells were transfected with Myc-Fbxl14b and Mkp3-HA together with increasing amounts of Flag-Fbxl14a $(0.6 \mu \mathrm{g}, 1.2 \mu \mathrm{g}, 2.4 \mu \mathrm{g})$. After $24 \mathrm{~h}$ of transfection, cells were treated with MG132 (10 $\mu \mathrm{M})$ for 6 $\mathrm{h}$ before harvesting. IP was performed using anti-Myc antibody and cell lysates and precipitated complexes were analyzed by western blotting using the indicated antibody. (L) Fbxl14a attenuates the polyubiquitination of Mkp3 induced by Fbxl14b. HEK293T cells were transfected as indicated for $24 \mathrm{~h}$ followed by treatment with MG132 $(10 \mu \mathrm{M})$ for $6 \mathrm{~h}$. Whole cell extracts were then processed for IP analysis of ubiquitinated Mkp3. Scale bar, $200 \mu \mathrm{m}$. 
of Mkp3, we co-transfected HEK293T cells with a vector encoding Mkp3-HA plus a vector encoding MycFbx114a, Myc-Fbx114b or Myc- $\Delta$ Fb (Supplementary information, Data S1). As shown in Figure 1E, the Mkp3 protein level was efficiently downregulated by the coexpression of wild-type Fbxl14b, whereas the $\Delta \mathrm{Fb}$ mutant could not negatively modulate Mkp3 level. In contrast, the level of Mkp3 was significantly increased by the coexpression of Fbx114a. As a major function of FBPs involves the ubiquitination of their substrate proteins, we sought to determine whether Fbx114a and Fbx114b regulate the ubiquitination of Mkp3 in mammalian cells. To this end, we co-transfected HEK293T cells with the constructs expressing Flag-Mkp3 and HA-tagged ubiquitin with Myc-Fbxl14a, Myc-Fbxl14b and Myc- $\Delta \mathrm{Fb}$, respectively. We then performed IP with an anti-Flag antibody. As shown in Figure 1F, the expression of Fbxl14b significantly enhanced the ubiquitination of Mkp3 compared with the empty vector control, $\Delta \mathrm{Fb}$ mutant and Fbxl14a. Furthermore, an in vitro ubiquitination assay showed that the ubiquitinated Mkp3 was dramatically increased by Fbxl14b but not by its F-box-deleted mutant, $\Delta \mathrm{Fb}$ (Figure 1G) (Supplementary information, Data S1). Moreover, the levels of ubiquitinated Mkp3 induced by Fbx114b were higher than those induced by Fbx114a. These results indicate that Fbxl14a and Fbxl14b act differently to regulate the ubiquitination and subsequently, the level of Mkp3. Importantly, the ectopic expression of $m k p 3$ could significantly rescue the dorsalized phenotype induced by fbxl14b (Figure 1H).

To test whether $f b x l 14 a$ and $f b x l 14 b$ might have a functional correlation in early embryonic patterning, we analyzed the phenotype of embryos in a series of coinjection experiments (Figure 1I). Embryos injected with mRNAs or MOs of the two genes exhibited an almost normal appearance. Embryos injected with $f b x l 14 b$ mRNA and $A$-MO displayed a dorsalized phenotype that resembled the phenotype of the $f b x l 14 b$ mRNAinjected embryos, whereas the phenotype caused by fbxll $4 a$ mRNA and $B$-MO co-injection was similar to the ventralization caused by $B-\mathrm{MO}$ injection alone. We then examined the expression patterns of the early markers of dorsal and ventral patterning (Figure 1J), which are consistent with their morphology. Considering that Fbx114a and Fbxl14b share a high degree of sequence homology, we then speculated that Fbx114a may negatively regulate the function of Fbxl14b through a competitive interaction with their common substrate, Mkp3. As expected, the interaction of Mkp3 with Fbxl14b was decreased in a dosedependent manner upon the expression of Fbx114a (Figure $1 \mathrm{~K})$. Consistently, we further showed that Fbxl14a can attenuate the ubiquitination of Mkp3 induced by Fbx114b
(Figure 1L).

In summary, based on gain- and loss-of-function experiments, we studied the biological function of two FBPs, Fbxl14a and Fbxl14b, during vertebrate embryonic DV axis formation. For the first time, we demonstrated that these FBPs have distinct functions: Fbxl14b exerts a strong dorsalizing activity, whereas Fbx114a functions to inhibit dorsalization. Our results suggest that Fbx114a and Fbx114b may act antagonistically, with Fbx114b having a more dominant effect, resulting in the overall activity of the two genes towards dorsalization. In addition, we identified Mkp3 as a novel binding partner of Fbxl14a and Fbxl14b. Mkp3 is required for proper axial patterning during embryogenesis [8], and in agreement with this report, our data further indicated that Fbxl14a and Fbxl14b exert their functions during axis formation through differentially modulating Mkp3 ubiquitination and protein levels.

Many E3 ligases, such as $\mathrm{SCF}^{\mathrm{Fbw} 7}$ and $\mathrm{SCF}^{\mathrm{Skp} 2}$, act as either tumor suppressor proteins or oncoproteins, depending on their ability to trigger the degradation of either oncoproteins or tumor suppressor proteins, respectively. A recent study reports that the expression of Fbx114 is markedly downregulated concomitantly with increased levels of its target Snaill protein during hypoxia, which has been associated with tumor progression [7]. Mkp3, a novel target of Fbxl14 in this study, is involved in the development of cancer and has been suggested as a candidate tumor suppressor protein [9]. Moreover, Mkp3 has also been correlated with chemoresistance of cancer cells $[10,11]$. Taken together, these findings indicate that Fbxl14 has the potential to play a role in cancer development, and they may help to elucidate the mechanism of Mkp3-related chemoresistance.

\section{Acknowledgments}

We thank Dr Ceshi Chen (The Kunming Institute of Zoology, Chinese Academy of Sciences, China) for kindly providing the rbxl and skpl vector. This work was supported by the National Basic Research Program of China (973 Program) (2011CB966301 and 2007CB947100), and the Shanghai Municipal Commission for Science and Technology (074319111 and 07DZ22919).

Hongping Zheng ${ }^{1}$, Yong Du ${ }^{1}$, Ye Hua ${ }^{1}$, Zhili Wu ${ }^{1}$, Yuanchang Yan $^{1}$, Yiping $\mathrm{Li}^{1}$

${ }^{I}$ State Key Laboratory of Cell Biology, Shanghai Key Laboratory for Molecular Andrology, Institute of Biochemistry and Cell Biology, Shanghai Institutes for Biological Sciences, Chinese Academy of Sciences, Shanghai 200031, China

Correspondence: Yiping Li

Tel: 86-21-54921413; Fax: 86-21-54921415

E-mail: Yipingli@sibs.ac.cn 


\section{References}

1 Ho MS, Ou C, Chan YR, Chien CT, Pi H. The utility F-box for protein destruction. Cell Mol Life Sci 2008; 65:1977-2000.

2 Nakayama KI, Nakayama K. Ubiquitin ligases: cell-cycle control and cancer. Nat Rev Cancer 2006; 6:369-381.

3 Nakayama KI, Nakayama K. Regulation of the cell cycle by SCF-type ubiquitin ligases. Semin Cell Dev Biol 2005; 16:323333.

4 Cardozo T, Pagano M. The SCF ubiquitin ligase: insights into a molecular machine. Nat Rev Mol Cell Biol 2004; 5:739-751.

5 Jin J, Cardozo T, Lovering RC, et al. Systematic analysis and nomenclature of mammalian F-box proteins. Genes Dev 2004; 18:2573-2580.

6 Vernon AE, LaBonne C. Slug stability is dynamically regulated during neural crest development by the F-box protein Ppa. Development 2006; 133:3359-3370.

7 Vinas-Castells R, Beltran M, Valls G, et al. The hypoxia-controlled FBXL14 ubiquitin ligase targets SNAIL1 for proteasome degradation. J Biol Chem 2010; 285:3794-3805.

8 Tsang M, Maegawa S, Kiang A, et al. A role for MKP3 in axial patterning of the zebrafish embryo. Development 2004; 131:2769-2779.

9 Wu GS. Role of mitogen-activated protein kinase phosphatases (MKPs) in cancer. Cancer Metastasis Rev 2007; 26:579-585.

10 Cui Y, Parra I, Zhang M, et al. Elevated expression of mitogen-activated protein kinase phosphatase 3 in breast tumors: a mechanism of tamoxifen resistance. Cancer Res 2006; 66:5950-5959.

11 Chan DW, Liu VW, Tsao GS, et al. Loss of MKP3 mediated by oxidative stress enhances tumorigenicity and chemoresistance of ovarian cancer cells. Carcinogenesis 2008; 29:17421750.

(Supplementary information is linked to the online version of the paper on the Cell Research website.) 